\title{
Method of Valve Preventive Maintenance via Additional Overview on POS for Decreasing Failure Risk at Starting and Change Over Fuel Gas to Fuel Oil in Block 1 and 2 Gas Turbine GT13E2 PT. PJB UP Muara Tawar
}

\author{
Alqa Nury $\mathrm{M}^{1}$, Seto Tri Sugianto ${ }^{1}$, Shafrudin Nurul Ihsan ${ }^{1}$, \\ Kevin Sanjoyo Gunawan ${ }^{2}$, Totok R. Biyanto ${ }^{2 *}$ \\ ${ }^{1}$ PT. PJB UP Muara Tawar, Muara Tawar, Indonesia \\ ${ }^{2}$ Engineering Physics Department, Institut Teknologi Sepuluh Nopember (ITS), Surabaya, Indonesia.
}

\begin{abstract}
Block 1 and 2 Combined Cycle Power Plant (CCPP) Muara Tawar have 5 units gas turbine can be operated by using fuel gas. Due to limitation of fuel gas from gas company. Several gas turbine are operated using High Speed Diesel fuel (HSD) to fulfil load demand. The equipment that used for fuel gas system must have high realibility to serve in dual fuel system neither operated using fuel oil or gas without unit trip. In actual, there are several disturbances occur during change over from gas to oil, even when the plant operated using fuel oil. From the data of failure causes, one of the failure causes is valve failure. By considering the availbility of plant, in term of possibility to modify instrumentation and control system, gas turbine 1.3 was choosen as object of the study. Additional indicator in HMI can be described as follows additional pressure switch attribute at EDS-P3 using symbol K, upload program to EPROM at card $70 B K 06$ as communication card, and the cycle time less than 1 second in both valve open or close. It indicate valve system under good condition or no maintenance action is required. Valve maintenance at gas turbine ALSTOM 13E2 in Block 1 and 2 CCPP Muara Tawar can be done by additional indicator of pressure switch and aux relay contact as feedback signal on POS 30. Therefore, no disturbances in valve system and gas turbine performance increases.
\end{abstract}

Keywords: failure risk, fuel gas, fuel oil, gas turbine, preventive maintenance

\section{Introduction}

Fuel gas as source of energy that is mostly used in industrial power plant [1]. Block 1 and 2 of CCPP Muara Tawar have 5 unit gas turbine which is operated using fuel gas. Fuel gas is supplied from gas company through pipe line [2]. Due to limitation of fuel gas from gas company. Several gas turbine are operated using High Speed Diesel fuel (HSD) to fulfil load demand [3,4]. The equipment that used for fuel gas system must have high realibility to serve in dual fuel system neither operated using fuel oil or gas without unit trip. In actual, there are several disturbances occur during change over from gas to oil, even when the plant operated using fuel oil. From the data of failure causes, one of the failure causes is valve failure. The valve failures are caused by leakage in membrane or line system, selenoid valve stucked, contactor and relay broken and fail in air and electric power supply [5]. Therefore, the preventive maintanance is required to overcome hidden failure in the valve by perform optimization of preventive maintenance [6,7].

\subsection{Sistem Sector Valve}

\section{Theory}

Gas turbine in CCPP Muara Tawar are ALSTOM type 13E2 that consist 18 valves to regulate air combustion at 6 area i.e. area MBN41, MBN42, and MBN43.

\subsection{P \& I Diagram (Pipping and Instrumentation Diagram)}

P\&I Diagram represent all equipment, instrumentation and interconnection all of them. The standard of this diagram based on International Society of Automation (ISA) Standard S5.1. P\&I Diagram is important document for operation, maintenance and process modification for operation and maintenance company, such as PT. PJB UP Muara Tawar. P\&ID is developed from process flow diagram (PFD) that describe mass and energy balances. This diagram describes actual or as build existing plant that will used for all engineering discipline to realize design into plant. Process engineer use this document to calculate size of equipment, safety engineer for HAZOP study, piping engineer for design piping and support, instrument engineer for design instrumentation such as transmitter, controller and valve. 


\section{Method}

\subsection{Fuel Oil Distribution on Gas Turbine Alstom 13E2}

As mentioned before, gas turbine CCPP Muara Tawar Blok 1 and 2 using Alstom type 13E2. Fuel oil distribution system in this turbine consist of 72 burner and 3 area control valve for fuel oil i.e. MBN41, MBN42, and MBN43. Distribution of 72 burners flow through six valves area. Each area serve some burner. For MBN41 serve to burner.MBN42 serve to for burner and MBN43 serve to six burner. P\&ID of fuel oil distribution system is shown in Figure 1 and 2.

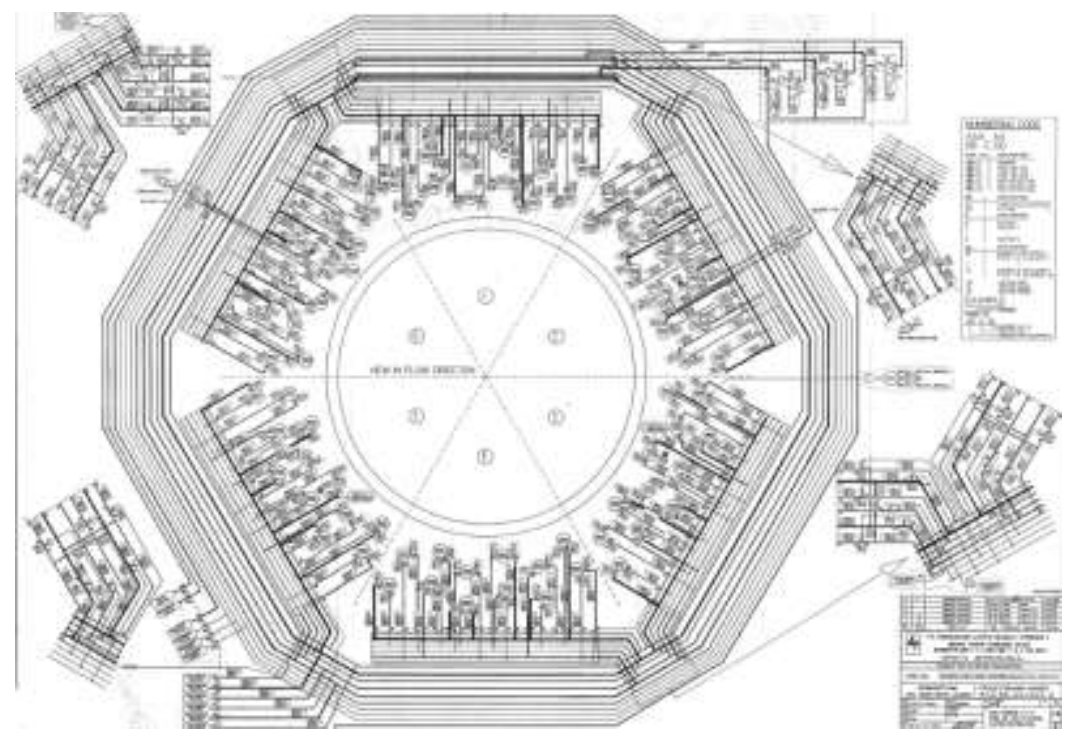

Figure 1. P\&I Diagram of Fuel Oil Distribution

Flow rate of fuel oil from storage tank to burner are controlled by using 18 valve areas. All the valves are actuated by air supply through 7 solenoid valves.

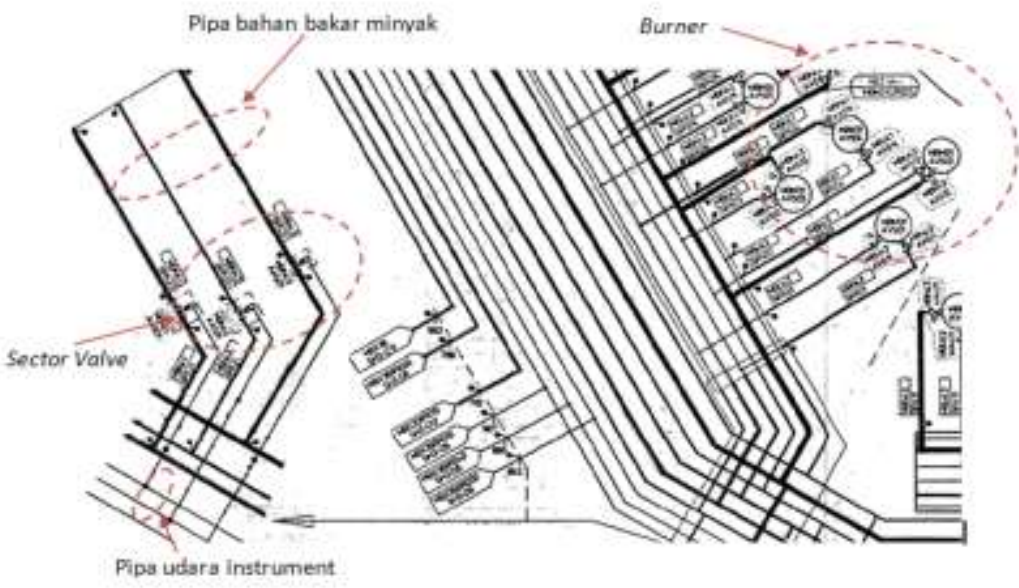

Figure 2. P\&I Diagram of Valve Area 5

Each solenoid valve consist of pressure switch lay at outlet of solenoid valve and serve an area valve with configuration as follows:

- Solenoid valve MBX36AA041 serve valve MBN41 for sector 2, 3, 5 and 6.

- Solenoid valve MBX36AA042 serve valve MBN42 for sector 2, 3, 5 dan 6.

- Solenoid valve MBX36AA043 serve valve MBN43 for all sector.

- Solenoid valve MBX36AA026 serve valve MBN41 for sector 1 (MBN41/101).

- Solenoid valve MBX36AA027 serve valve MBN41 for sector 4 (MBN41/401).

- Solenoid valve MBX36AA028 serve valve MBN42 for sector 1 (MBN42/101).

- Solenoid valve MBX36AA029 serve valve MBN42 for sector 4 (MBN42/401). 


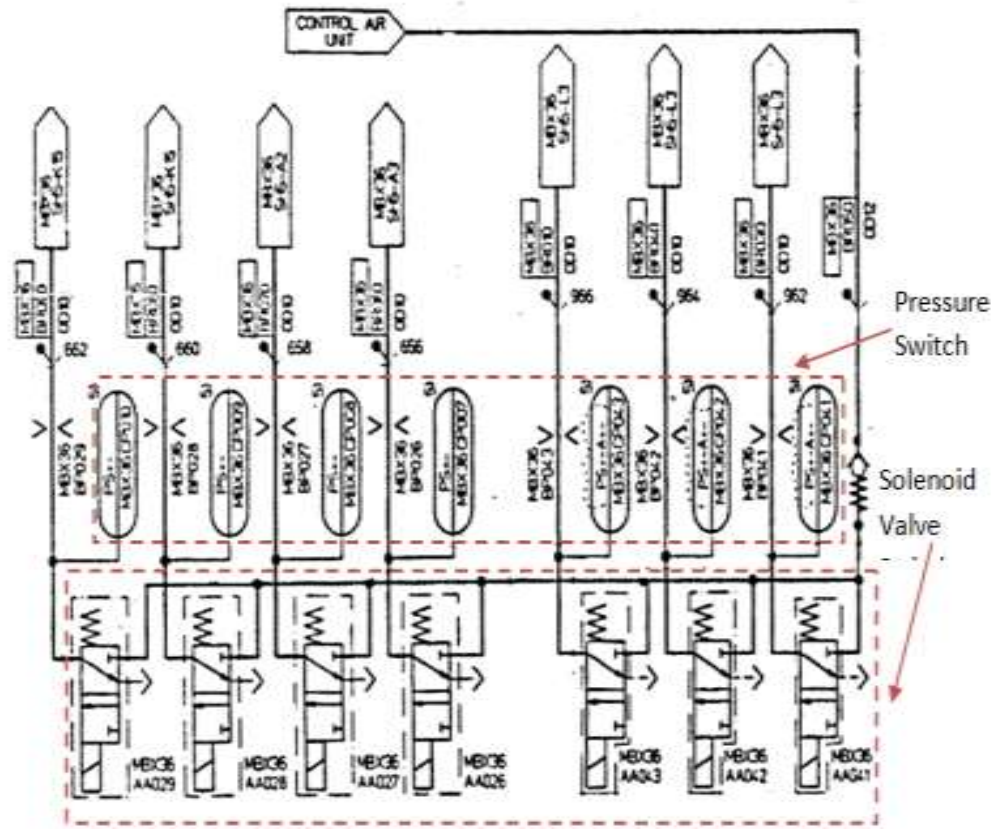

Figure 3. P\&I Diagram Solenoid Valve and Pressure Switch in Sector Valve

\subsection{Mapping System on Valve}

Valve consist of valve body, actuator and sensor that operate normally open or air to close. In actuator, there are some part with function as follows:

- Card 70AB02 as an output modul that transmitt voltage signal $24 \mathrm{VDC}$ to interposing relay, if output from PLC states $=1$.

- Interposing relay convert $24 \mathrm{VDC}$ to $220 \mathrm{VAC}$ to activate contactor coil.

- Contactor is used to energize the solenoid coil with $220 \mathrm{VAC}$

- Solenoid valve is utilized to supply pneumatic valve with normally close or energize to open. Sensor consist of:

- Pressure switch detect air pressurer that flow into the valve. pressure switch setting open at pressure system above 4 bar and vice versa.

- Aux relay detect the states of valve position

- Card 70EB02 as a converted modul that change switch position of pressure switch and aux relay become binary input signal to the PLC.

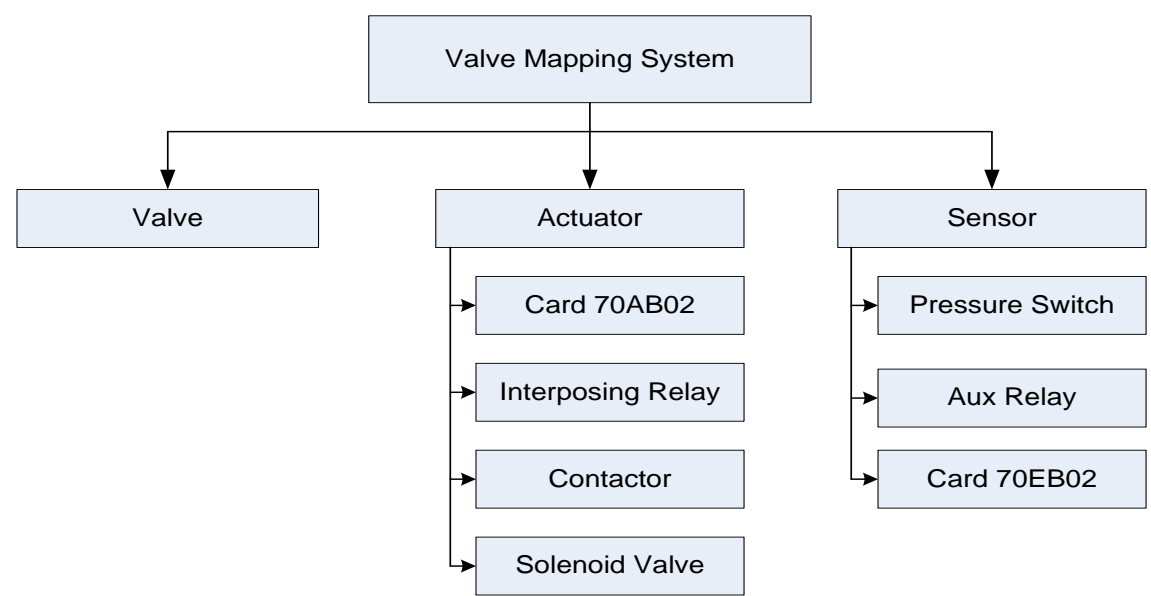

Figure 4. Mapping on Gas Turbine Valve 


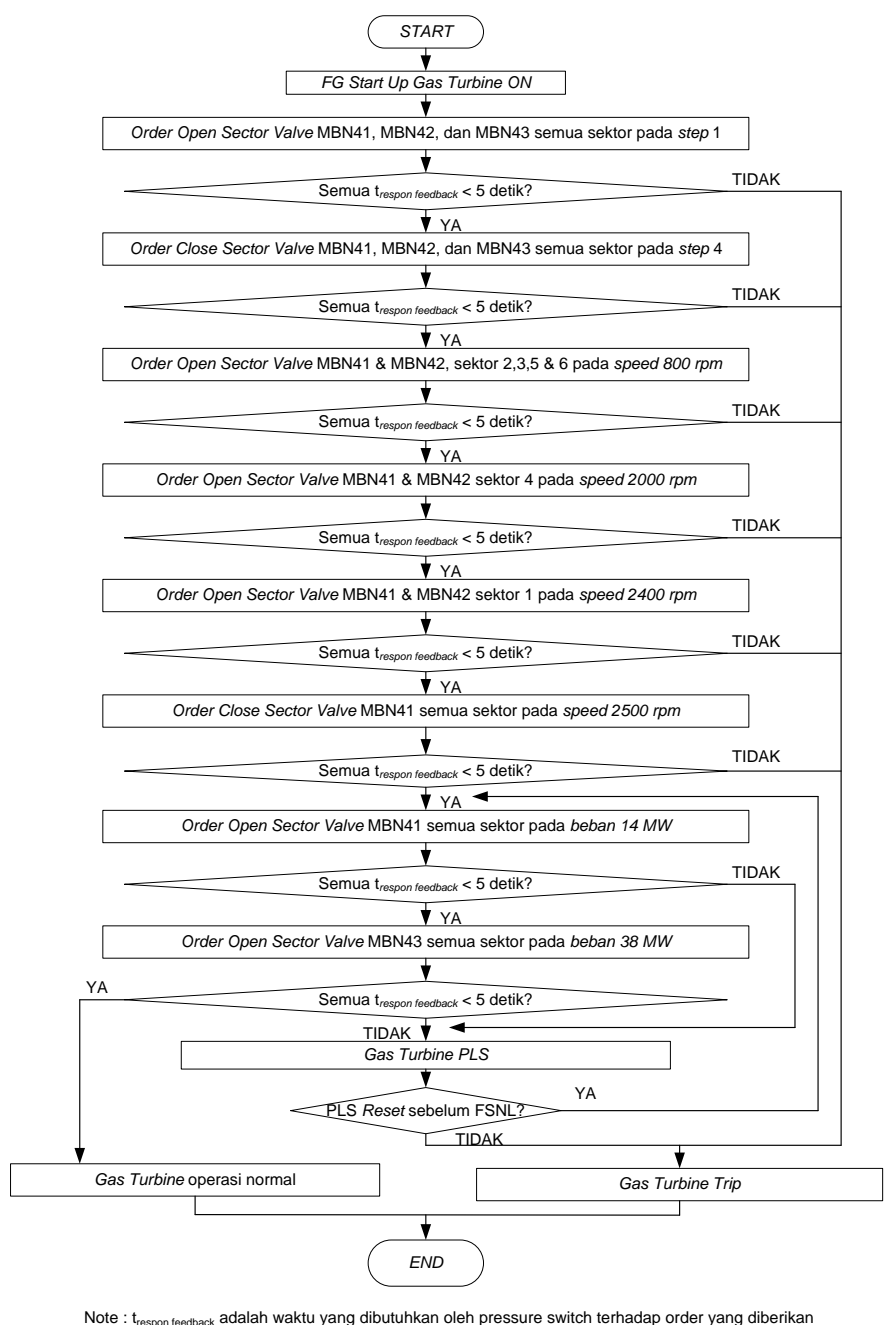

Figure 5. Process Maping Sector Valve When Start Up Turbine Gas

\subsection{Gas Turbine Protection}

Protection system in the gas turbin to avoid valve wrong position are shown in Figure 6 and 7.

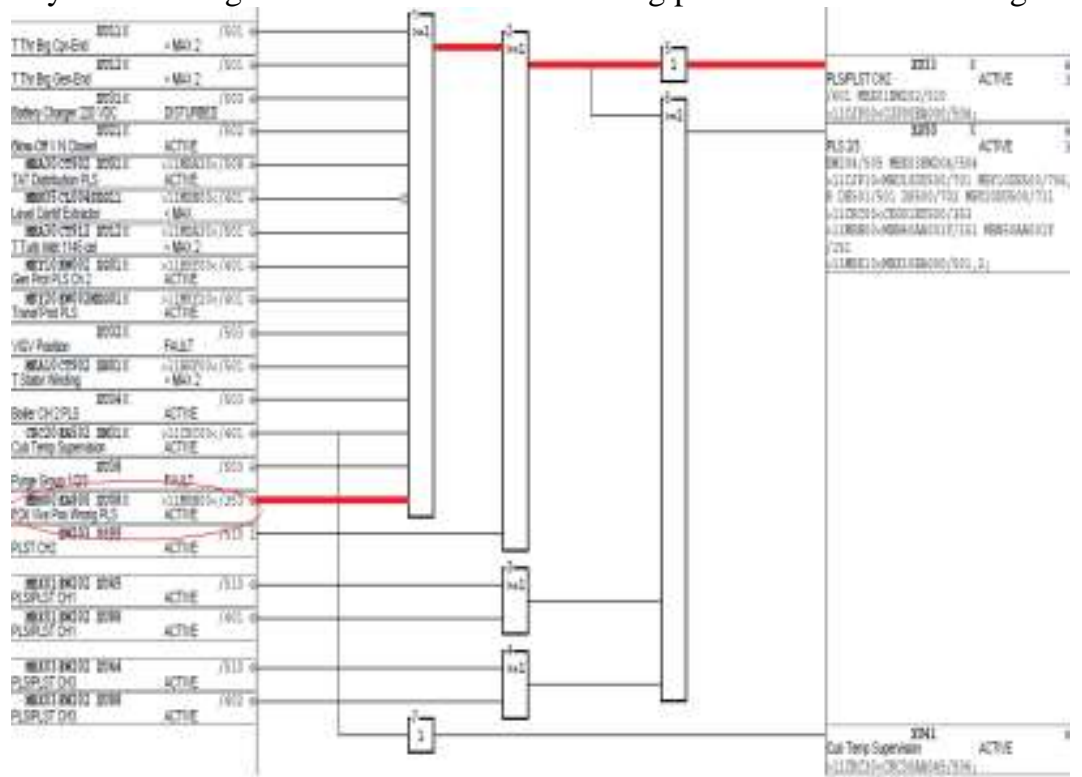

Figure 6. Logic Diagran Plst for Fuel Oil Wrong Position 


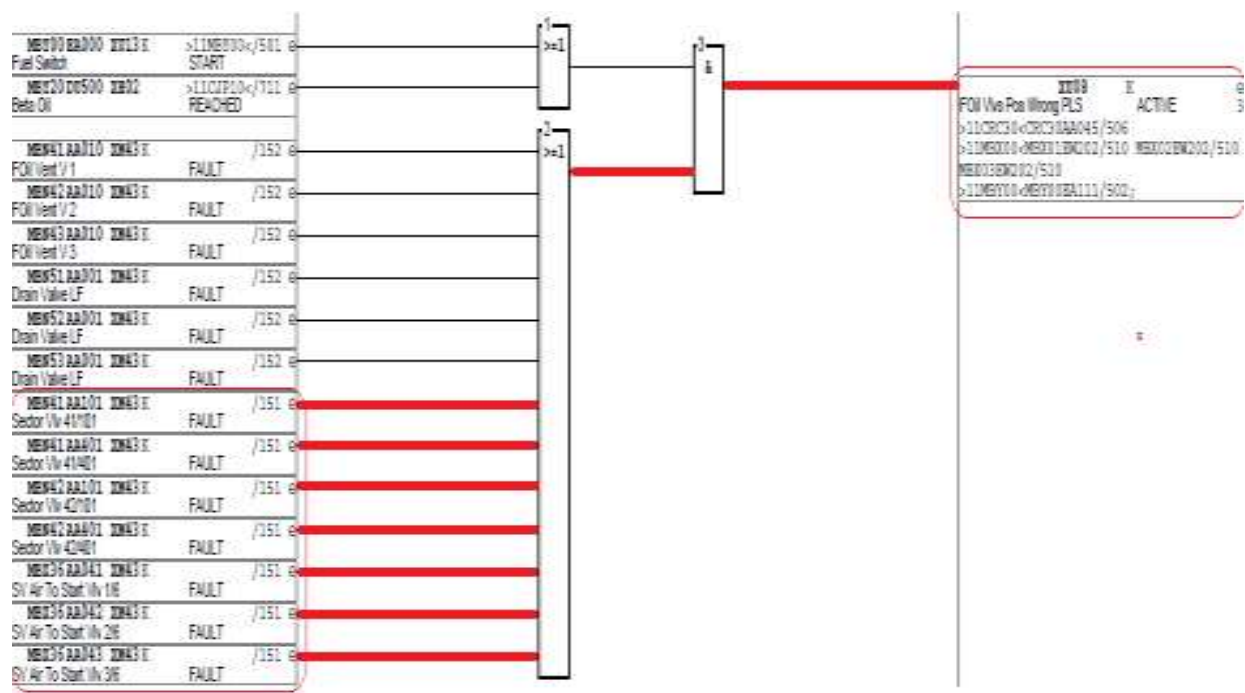

Figure 7. Logic Diagram Fuel Oil Wrong Position in Valve

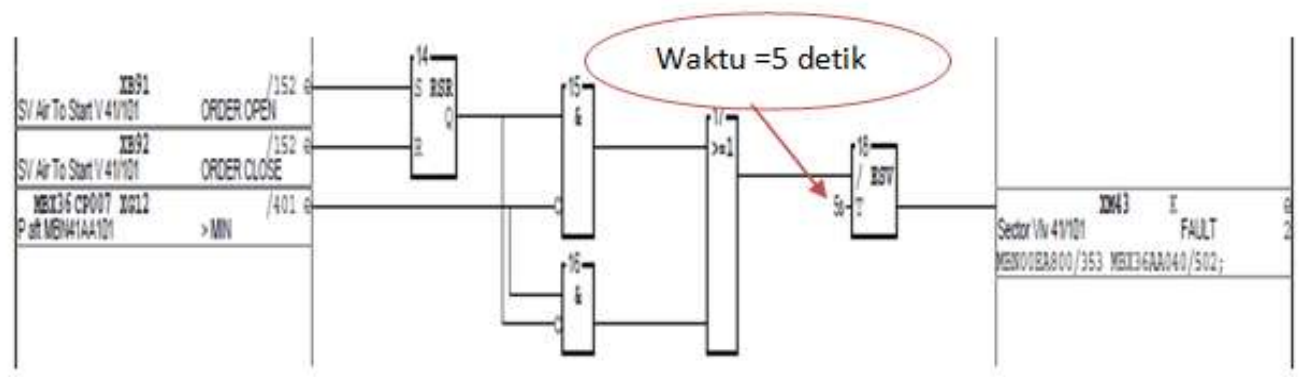

Figure 8. Logic Diagram Valve MBN41AA101

\subsection{Valve Overview POS30}

Human Machine Interface (HMI) CCPP Muara Tawar uses POS30 as an interface between operator and plant system. The status of valve both open or close can be viewed in HMI based on feedback signal from auxillary contactor, it is shown in Figure 9.

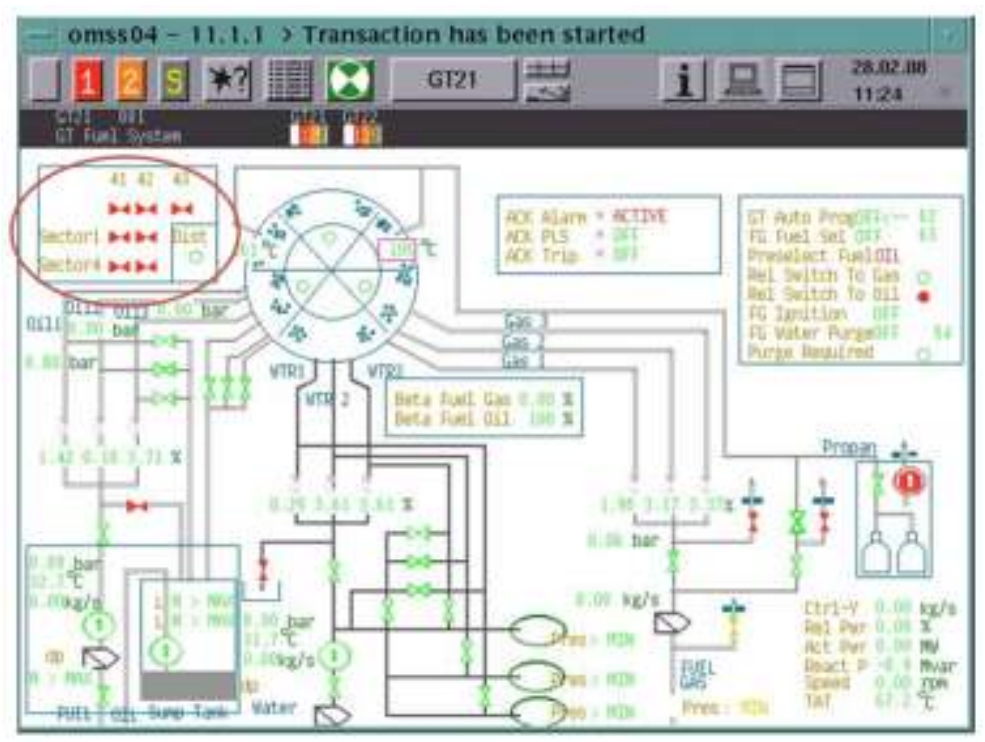

Figure 9. Overview Valve and Disturbed Indication 
Method of Valve Preventive Maintenance via Additional Overview on POS for Decreasing Failure

\subsection{Problem Solving}

Table 1 shows the causes, failures and actions in gas turbine during actual operation.

Table 1. Failure in Gas Turbine.

\begin{tabular}{|c|c|c|c|c|}
\hline & Date & Disturbance & Cause & Improvement \\
\hline 1 & 13 August 2007 & $\begin{array}{l}\text { Start Failure of SV MBN41 / } 101 \\
\text { Failed }\end{array}$ & $\begin{array}{l}\text { etting pressure switch not in } 4 \\
\text { bars }\end{array}$ & $\begin{array}{l}\text { Replace and calibrate pressure } \\
\text { switch } M B N 41 / 101\end{array}$ \\
\hline 2 & 04 June 2008 & $\begin{array}{l}\text { Start Failure of SV MBN42 / } 101 \\
\text { Failed }\end{array}$ & $\begin{array}{l}\text { Movement of solenoid valve } \\
\text { more than } 5 \text { seconds }\end{array}$ & Cleaning SV MBN42 / 101 \\
\hline 3 & 07 January 2008 & $\begin{array}{l}\text { Start Failure from SV MBN41/401 } \\
\text { Failed }\end{array}$ & $\begin{array}{l}\text { Movement of solenoid valve } \\
\text { more than } 5 \text { seconds }\end{array}$ & Cleaning all SV Sector Valve \\
\hline 4 & 08 January2008 & $\begin{array}{l}\text { Start Failure from SV MBN42/401 } \\
\text { Failed }\end{array}$ & $\begin{array}{l}\text { Movement of solenoid valve } \\
\text { more than } 5 \text { seconds }\end{array}$ & $\begin{array}{l}\text { Test open close all SV sector } \\
\text { valve }\end{array}$ \\
\hline 5 & 15 March 2009 & $\begin{array}{l}\text { Change Over Fuel Failure from SV } \\
\text { Air To Start MBN42 Failed }\end{array}$ & $\begin{array}{l}\text { Movement of solenoid valve } \\
\text { more than } 5 \text { seconds }\end{array}$ & Cleaning all SV Sector Valve \\
\hline 6 & 14 March 2009 & $\begin{array}{l}\text { Change Over Fuel Failure from SV } \\
\text { Air To Start MBN41 Failed }\end{array}$ & $\begin{array}{l}\text { Movement of solenoid valve } \\
\text { more than } 5 \text { seconds }\end{array}$ & Cleaning all SV Sector Valve \\
\hline 7 & 14 March 2009 & PLST from $S V M B N 41 / 101$ Failed & $\begin{array}{l}\text { Movement of solenoid valve } \\
\text { more than } 5 \text { seconds }\end{array}$ & Cleaning all SV Sector Valve \\
\hline 8 & $\begin{array}{ll}21 & \text { November } \\
2010 & \\
\end{array}$ & $\begin{array}{l}\text { Start Failure from SV Air To Start } \\
\text { MBN41 Failed }\end{array}$ & $\begin{array}{l}\text { Movement of solenoid valve } \\
\text { more than } 5 \text { seconds }\end{array}$ & $\begin{array}{lllll}\text { Cleaning } & \text { SV } & \text { Air } & \text { To } & \text { Start } \\
\text { MBN41 } & & & & \\
\end{array}$ \\
\hline 9 & 13 January 2010 & $\begin{array}{l}\text { Start Failure from SV Air To Start } \\
\text { MBN43 Failed }\end{array}$ & $\begin{array}{l}\text { Setting pressure switch not in } 4 \\
\text { bars }\end{array}$ & $\begin{array}{l}\text { Replace and calibrate pressure } \\
\text { switch Air To Start MBN43 }\end{array}$ \\
\hline 10 & 08 August 2010 & PLST from $S V M B N 42 / 401$ Failed & Failed Contactor & $\begin{array}{lll}\begin{array}{l}\text { Replace } \\
\text { contactor }\end{array} & \text { SV } & \text { MBN41/101 } \\
\end{array}$ \\
\hline 11 & $\begin{array}{ll}24 & \text { December } \\
2010 & \\
\end{array}$ & $\begin{array}{l}\text { Start Failure from SV Air Tor Start } \\
\text { MBN41 Failed }\end{array}$ & $\begin{array}{l}\text { Movement of solenoid valve } \\
\text { more than } 5 \text { seconds }\end{array}$ & Cleaning all SV Sector Valve \\
\hline 12 & $\begin{array}{ll}25 & \text { December } \\
2010 & \end{array}$ & $\begin{array}{l}\text { Start Failure from SV Air Tor Start } \\
\text { MBN41 Failed }\end{array}$ & $\begin{array}{l}\text { Movement of solenoid valve } \\
\text { more than } 5 \text { seconds }\end{array}$ & $\begin{array}{lllll}\begin{array}{l}\text { Cleaning } \\
\text { MBN41 }\end{array} & \text { SV } & \text { Air } & \text { To } & \text { Start } \\
\end{array}$ \\
\hline 13 & 13 January 2010 & $\begin{array}{l}\text { Start Failure from SV Air To Start } \\
\text { MBN42 Failed }\end{array}$ & $\begin{array}{l}\text { Setting pressure switch not in } 4 \\
\text { bars }\end{array}$ & $\begin{array}{l}\text { Replace and calibrate pressure } \\
\text { switch Air To Start MBN42 }\end{array}$ \\
\hline 14 & 14 January 2010 & $\begin{array}{l}\text { Start Failure from SV MBN42/401 } \\
\text { Failed }\end{array}$ & $\begin{array}{l}\text { Movement of solenoid valve } \\
\text { more than } 5 \text { seconds }\end{array}$ & $\begin{array}{l}\text { Test open close all SV sector } \\
\text { valve }\end{array}$ \\
\hline 15 & 24 April 2010 & $\begin{array}{l}\text { Start Failure from SV MBN42/401 } \\
\text { Failed }\end{array}$ & $\begin{array}{l}\text { Movement of solenoid valve } \\
\text { more than } 5 \text { seconds }\end{array}$ & Cleaning SV MBN42/401 \\
\hline 16 & 16 May 2011 & $\begin{array}{l}\text { Start Failure from SV MBN41/401 } \\
\text { Failed }\end{array}$ & $\begin{array}{l}\text { Movement of solenoid valve } \\
\text { more than } 5 \text { seconds }\end{array}$ & Cleaning all SV Sector Valve \\
\hline 17 & 20 May 2011 & $\begin{array}{l}\text { Start Failure from SV Air To Start } \\
\text { MBN41 Failed }\end{array}$ & $\begin{array}{l}\text { Movement of solenoid valve } \\
\text { more than } 5 \text { seconds }\end{array}$ & Cleaning all SV Sector Valve \\
\hline 18 & 05 January 2012 & $\begin{array}{l}\text { Start Failure from SV MBN42/401 } \\
\text { Failed }\end{array}$ & $\begin{array}{l}\text { Movement of solenoid valve } \\
\text { more than } 5 \text { seconds }\end{array}$ & Cleaning all SV Sector Valve \\
\hline 19 & 17 February 2012 & $\begin{array}{l}\text { Start Failure from SV MBN42/401 } \\
\text { Failed }\end{array}$ & Failed Contactor & $\begin{array}{l}\text { replace } \\
\text { contactor }\end{array}$ \\
\hline
\end{tabular}

Data in Table 1 can be presented as pareto chart as shown in Fig.10.

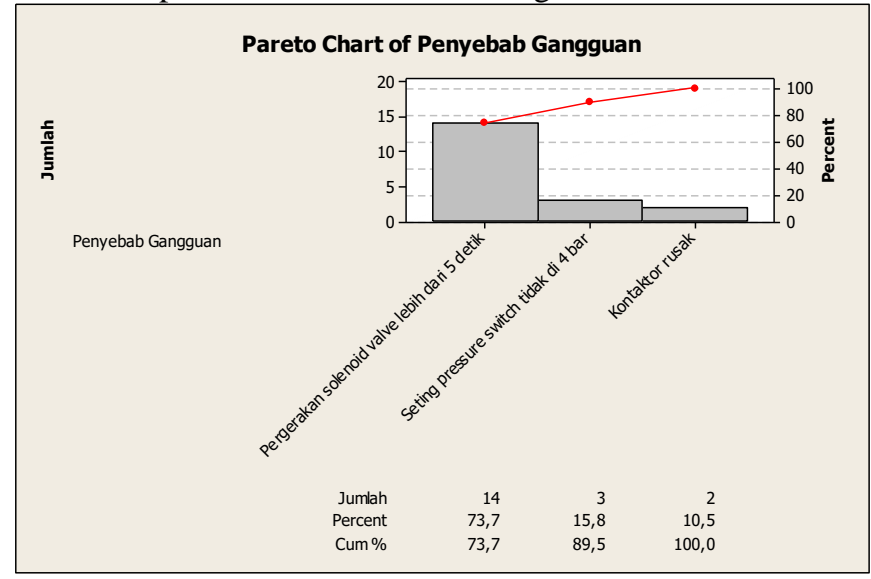

Figure 10. Pareto Chart Valve Failure

Based on pareto chart, the biggest percented of gas turbine valve failure about $73,7 \%$ due to slow respon at solenoid valve more than 5 second. The solution of this problem to avoid slow respon more than 5 second are tabuleted in Table 2 
Table 2. Solution of Problem

\begin{tabular}{|l|l|l|}
\hline Problem Cause & Solution & Note \\
\hline There are some impurities in solenoid valve & Cleaning pilot solenoid & $\begin{array}{l}\text { Preventive } \\
\text { maintenance done }\end{array}$ \\
\hline There are no method to monitor solenoid valve & $\begin{array}{l}\text { additional overview POS 30 for pressure } \\
\text { switch as feedback in aux relay contactor }\end{array}$ & \\
\hline Air instrument contain water & Installation of drain instrument & \\
\hline $\begin{array}{l}\text { Feedback pressure switch condition not yet } \\
\text { shown in POS30 }\end{array}$ & Showing pressure switch di POS30 & \\
\hline
\end{tabular}

Refer to Table 2, the required action to overcome the problem can be describe as follows:

- Additional indicator in HMI using POS 30 and pressure switch and aux relay contact as feedback signal.

- Update maintenance task refer to existing instrumentation system especially valve in gas turbine system

\section{Results And Discussions}

By considering the availbility of plant, in term of possibility to modify instrumentation control system, gas turbine 1.3 was choosen as object of the study. Additional indicator in HMI can be described as follows:

1. Additional pressure switch attribute at EDS-P3 using symbol K

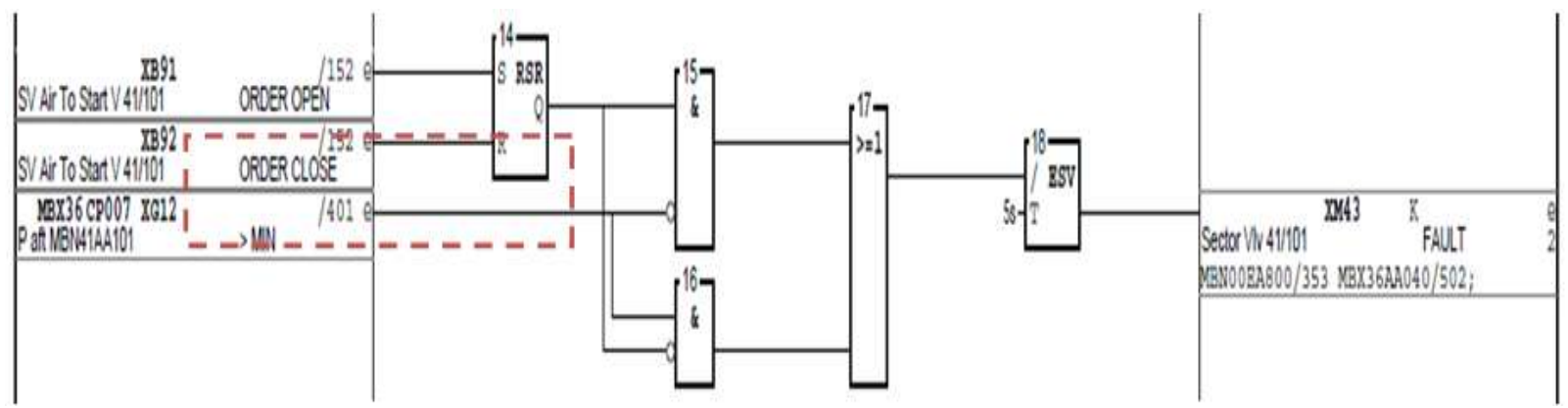

Figure 11. Pressure Switch Signal Before K Symbol Addition

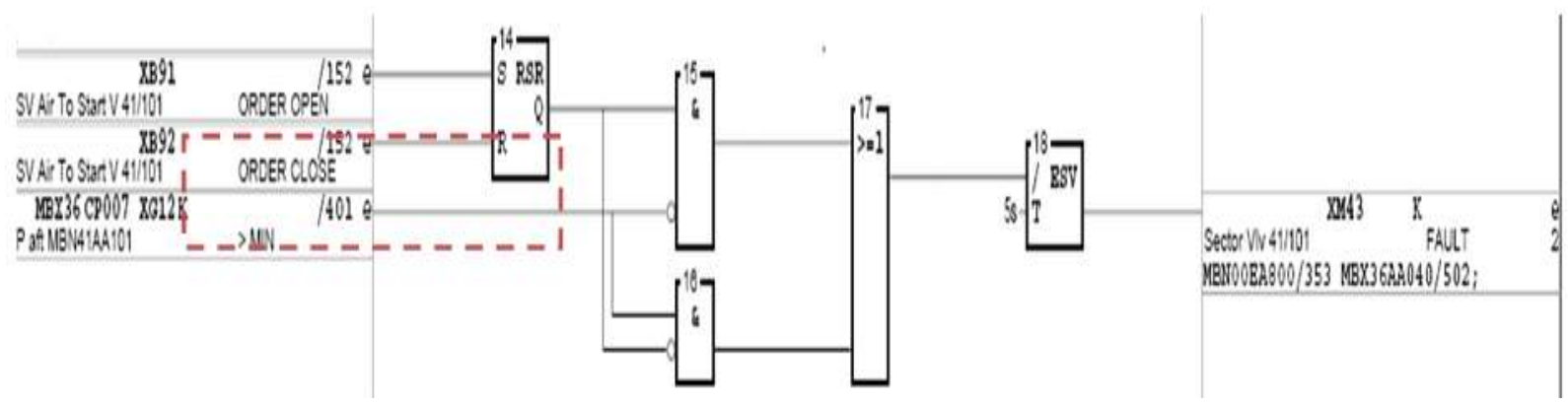

Figure 12. Pressure Switch Signal After K Symbol Addition

2. Upload program to EPROM at card 70BK06 as communication card

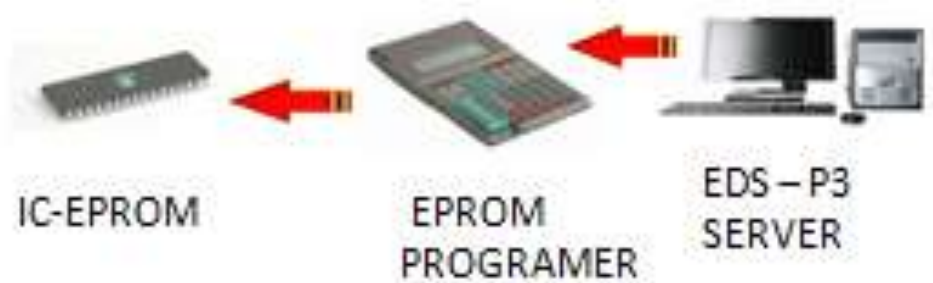

Figure 13. EPROM from EDS-P3

3. Address configuration on OPC server and OPC client

4. Build logic diagram on POS 30 Server 


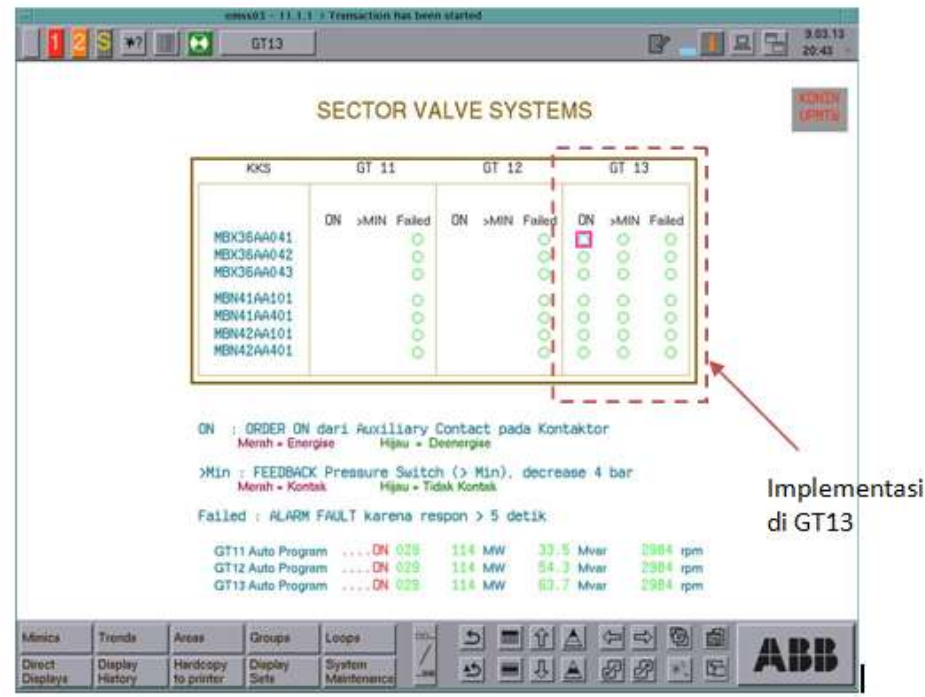

Figure 14. Overview System Valve Block 1

Testing of proposed design should be perform to evaluated the performance or cycle time of the system. Detail results of testing as shown in Fig. 15 and Table 3.

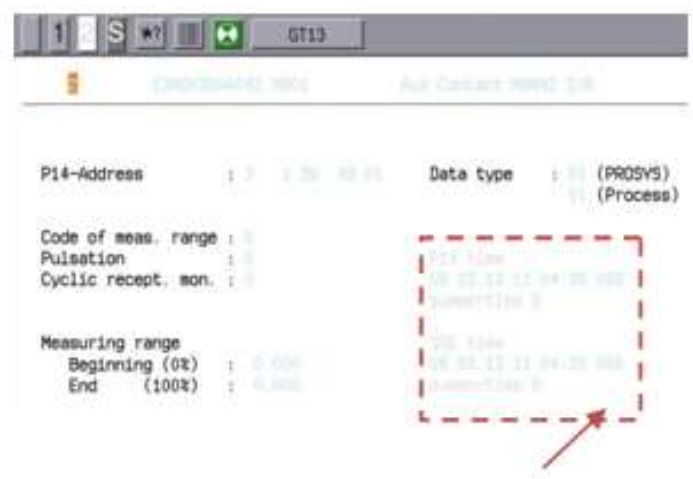

Waktu Aux contact

$13 \mathrm{MB} \times 36 \mathrm{AA0} 42$

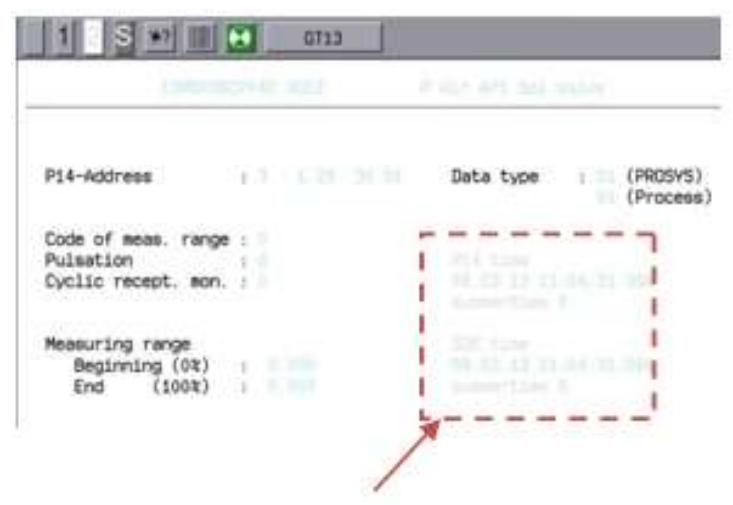

Waktu pressure switch

$13 \mathrm{MBX} 36 \mathrm{CP} 042$

Figure 15. Aux Contact Time and Pressure Switch in 13MBX36AA042 
Table 3. Response Time Pressure Switch by Aux Contact

\begin{tabular}{|c|c|c|c|}
\hline \multirow{2}{*}{ KKS } & \multicolumn{2}{|c|}{ Solenoid Valve (ORDER CLOSE) } \\
\cline { 2 - 4 } & $\begin{array}{c}\text { Aux Contact } \\
\text { (Energize Time) }\end{array}$ & $\begin{array}{c}\text { Pressure Switch } \\
\text { (Contact Time) }\end{array}$ & $\begin{array}{c}\text { Respond Time } \\
\text { (second) }\end{array}$ \\
\hline 13MBX36AA041 & $11: 11: 02$ & $11: 11: 02$ & 0 \\
\hline 13MBX36AA042 & $11: 11: 02$ & $11: 11: 02$ & 0 \\
\hline 13MBX36AA043 & $11: 11: 02$ & $11: 11: 02$ & 0 \\
\hline 13MBX41AA101 & $11: 11: 02$ & $11: 11: 02$ & 0 \\
\hline 13MBX41AA401 & $11: 11: 02$ & $11: 11: 02$ & 0 \\
\hline 13MBX42AA101 & $11: 11: 02$ & $11: 11: 02$ & 0 \\
\hline 13MBX42AA401 & $11: 11: 02$ & $11: 11: 02$ & 0 \\
\hline
\end{tabular}

Table 3. Response Time Pressure Switch by Aux Contact (Cont)

\begin{tabular}{|c|c|c|c|}
\hline \multirow{2}{*}{ KKS } & \multicolumn{3}{|c|}{ Solenoid Valve (ORDER OPEN) } \\
\cline { 2 - 4 } & $\begin{array}{c}\text { Aux Contact } \\
\text { (De-energize Time) }\end{array}$ & $\begin{array}{c}\text { Pressure Switch } \\
\text { (Open Time) }\end{array}$ & $\begin{array}{c}\text { Respond Time } \\
\text { (second) }\end{array}$ \\
\hline 13MBX36AA041 & $11: 04: 29$ & $11: 04: 29$ & 0 \\
\hline 13MBX36AA042 & $11: 04: 30$ & $11: 04: 31$ & 1 \\
\hline 13MBX36AA043 & $11: 04: 31$ & $11: 04: 31$ & 0 \\
\hline 13MBX41AA101 & $11: 04: 35$ & $11: 04: 36$ & 1 \\
\hline 13MBX41AA401 & $11: 04: 36$ & $11: 04: 37$ & 1 \\
\hline 13MBX42AA101 & $11: 04: 38$ & $11: 04: 39$ & 1 \\
\hline 13MBX42AA401 & $11: 04: 39$ & $11: 04: 40$ & 1 \\
\hline
\end{tabular}

Table 3 shows the cycle time less than 1 second in both valve open or close. It indicate valve system under good condition or no maintenance action is required

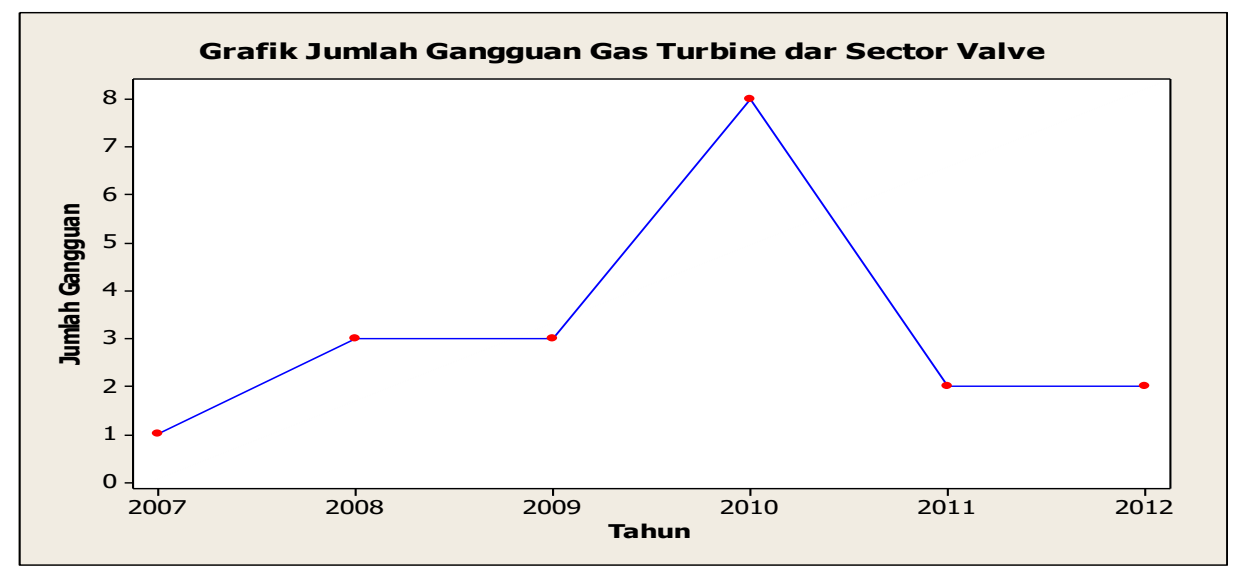

Figure 16. Valve Problem in 2007 - 2012 Period

Table 4. Problem in Valve

\begin{tabular}{|c|l|c|c|c|l|}
\hline \multicolumn{1}{|c|}{ Tanggal } & Loss (Hour) & $\begin{array}{c}\text { Loss Product } \\
\text { (MW) }\end{array}$ & Unit & \multicolumn{1}{|c|}{ Problem } \\
\hline $\mathbf{1}$ & 13 August 2007 & 2,27 & 306,0 & GT12 & Start Failure SV MBN41/101 Failed \\
\hline $\mathbf{2}$ & 04 Juny 2008 & 1,90 & 256,5 & GT12 & Start Failure from SV MBN42/101 Failed \\
\hline $\mathbf{3}$ & 07 January 2008 & 1,47 & 198,0 & GT21 & Start Failure from SV MBN41/401 Failed \\
\hline $\mathbf{4}$ & 08 January 2008 & 1,20 & 162,0 & GT22 & Start Failure from SV MBN42/401 Failed \\
\hline $\mathbf{5}$ & 15 March 2009 & 0,00 & 0,0 & GT11 & Change Over Fuel Failure from SV Air To Start MBN42 Failed \\
\hline $\mathbf{6}$ & 14 March 2009 & 0,00 & 0,0 & GT13 & Change Over Fuel Failure from SV Air To Start MBN41 Failed \\
\hline $\mathbf{7}$ & 14 March 2009 & 0,00 & 0,0 & GT13 & PLST from SV MBN 41/101 Failed \\
\hline $\mathbf{8}$ & 21 November 2010 & 2,78 & 389,7 & GT12 & Start Failure from SV Air To Start MBN41 Failed \\
\hline $\mathbf{9}$ & 13 January 2010 & 1,20 & 162,0 & GT21 & Start Failure from SV Air To Start MBN43 Failed \\
\hline $\mathbf{1 0}$ & 08 August 2010 & 3,73 & 541,3 & GT21 & PLST from SV MBN42/401 Failed \\
\hline $\mathbf{1 1}$ & 24 December 2010 & 0,37 & 51,3 & GT21 & Start Failure from SV Air Tor Start MBN41 Failed \\
\hline $\mathbf{1 2}$ & 25 December 2010 & 5,72 & 600,3 & GT21 & Start Failure from SV Air Tor Start MBN41 Failed \\
\hline $\mathbf{1 3}$ & 13 January 2010 & 4,52 & 609,8 & GT22 & Start Failure from SV Air To Start MBN42 Failed \\
\hline $\mathbf{1 4}$ & 14 January 2010 & 1,88 & 254,3 & GT22 & Start Failure from SV MBN42/401 Failed \\
\hline $\mathbf{1 5}$ & 24 April 2010 & 0,93 & 130,7 & GT22 & Start Failure from SV MBN42/401 Failed \\
\hline $\mathbf{1 6}$ & 16 May 2011 & 1,43 & 137,0 & GT21 & Start Failure from SV MBN41/401 Failed \\
\hline $\mathbf{1 7}$ & 20 May 2011 & 4,12 & 137,0 & GT21 & Start Failure from SV Air To Start MBN41 Failed \\
\hline $\mathbf{1 8}$ & 05 January 2012 & 1,02 & 137,7 & GT22 & Start Failure from SV MBN42/401 Failed \\
\hline $\mathbf{1 9}$ & 17 Februari 2012 & 1,73 & 233,6 & GT22 & Start Failure from SV MBN42/401 Failed \\
\hline
\end{tabular}




\section{Conclusion}

Valve maintenance at gas turbine ALSTOM 13E2 in Block 1 and 2 CCPP Muara Tawar can be done by additional indicator of pressure switch and aux relay contact as feedback signal on POS 30 . Therefore, no disturbances in valve system and gas turbine performance increases. .

\section{Acknowledgement}

The authors gratefully thank to PT. PJB UP Muara Tawar - Indonesia for providing the facilities in conducting this research.

\section{References}

[1]. A. Sieminski, "International Energy Outlook," US Energy Information Administration, Washington DC, 2013.

[2]. J. G. Speight, Natural Gas-A Basic Handbook, Texas, USA: Gulf Publishing Company, 2007.

[3]. R. J. Basavaraja and S. Jayanti, "Viability of fuel switching of a gas-fired power plant operating in chemical looping combustion mode," Energy, vol. 81, pp. 213-221, 2015.

[4]. G. Varympopiotis, A. Tolis and A. Rentizelas, "Fuel switching in power-plants: Modelling and impact on the analysis of energy projects," Energy Conversion and Management, vol. 77, pp. 650-667, 2014.

[5]. Emerson Process Management, Control Valve Handbook, Iowa: Fisher Control International, 2005.

[6]. A. M. Razak, Industrial gas turbines performance and operability, Cambridge: Woodhead Publishing Limited, 2007.

[7]. M. Naeem, R. Singh and D. Probert, "Implications of engine deterioration for creep life," Applied Energy, vol. 60, pp. 183-223, 1998. 